Forum 2020 · 35:267

https://doi.org/10.1007/s12312-020-00827-7

Online publiziert: 15. Juli 2020

(c) Springer Medizin Verlag GmbH, ein Teil von

Springer Nature 2020

Deutsche Krebsgesellschaft e.V.

Berlin, Deutschland

\title{
Finanzierung der ambulanten Krebsberatung weiterhin nicht gesichert
}

der psychosozialen Beratungsleistungen muss deshalb in ganz Deutschland gesichert sein. Neben dem nun gesicherten Anteil der GKV brauchen wir einen Einstieg der Rentenversicherung in die Finanzierung der Krebsberatungsstellen. Dafür ist eine gesetzliche Initiative des Bundesarbeitsministeriums dringend notwendig", sagt Dr. Johannes Bruns, Generalsekretär der DKG.

\section{》K Krebspatientinnen und -patienten benötigen eine flächendeckend einheitliche psychosoziale Unterstützung}

Die DKG begrüßt, dass durch die Teilfinanzierung der Kassen ein erster Schritt hin zur Kostendeckung und Qualitätsentwicklung in der Krebsberatung erfolgt. Voraussetzungen für die Finanzierung der Beratung bei psychischen Belastungen sind bestimmte Qualitätsanforderungen, die von Beratungsstellen erfüllt werden müssen. Die etwa 130 Krebsberatungsstellen der Landeskrebsgesellschaften seien diesbezüglich gut aufgestellt, so Bruns. Die Deckung der Kosten für eine sozialrechtliche Hilfestellung beruht aktuell auf Spendenmittel, projektbezogenen Förderungen und freiwilligen Zahlungen verschiedener Kostenträger, unter anderem der Rentenversicherung und der Länder. Nicht alle Beratungsstellen der Landeskrebsgesellschaften haben die Teilfinanzierung der übrigen Kosten für die sozialrechtliche Beratung bisher sichern können. Um eine dauerhafte Versorgung an Krebs erkrankter Personen mit psychosozialen Beratungen flächendeckend sicherzustellen, ist eine umfängliche Regelfinanzierung unerlässlich.
Krebsberatungsstellen der Landeskrebsgesellschaften sind für Patient ${ }^{*}$ innen eine wichtige und niedrigschwellige Anlaufstelle bei psychischen Belastungen und sozialrechtlichen Fragestellungen, die im gesamten Verlauf einer Krebserkrankung auftreten können. Voraussetzung für eine Förderung durch die GKV sind Qualitätsanforderungen, etwa die Qualifikation des Personals, räumliche Voraussetzungen, das EDV-gestützte Qualitätsmanagement und die Dokumentationen der Fälle.

\section{Korrespondenzadresse}

Angelina Gromes

Pressestelle der Deutschen

Krebsgesellschaft e. V.

gromes@krebsgesellschaft.de

\section{Katrin Mugele}

mugele@krebsgesellschaft.de

www.krebsgesellschaft.de 